\title{
The physician-patient relationship in the age of precision medicine
}

\author{
Gil Eyal, $\mathrm{PhD}^{1}$, Maya Sabatello, $\mathrm{LLB}, \mathrm{PhD}^{1}$, Kathryn Tabb, $\mathrm{PhD}^{1}$, Rachel Adams, $\mathrm{PhD}^{1}$, \\ Matthew Jones, $\mathrm{PhD}^{1}$, Frank R. Lichtenberg, $\mathrm{PhD}^{1}$, Alondra Nelson, $\mathrm{PhD}^{1}$, Kevin Ochsner, $\mathrm{PhD}^{1}$, \\ John Rowe, MD ${ }^{1}$, Deborah Stiles, JD ${ }^{1}$, Kavita Sivaramakrishnan, PhD ${ }^{1}$, \\ Kristen Underhill, DPhil, JD ${ }^{1}$ and Paul S. Appelbaum, MD ${ }^{1}$
}

Genetics in Medicine (2019) 21:813-815; https://doi.org/10.1038/s41436-018-0286-z

The completion of the Human Genome Project was heralded as a step towards "personalized medicine," offering patients individualized treatments based on genomic profiling. More recently, this vision has been eclipsed by the promise of "precision medicine" (PM), emphasizing benefits to patients from more precise diagnosis and treatment based on a range of biomarkers, along with data about patients' environment, lifestyle, and behaviors. ${ }^{1}$

Cynics may object that PM is mostly hype and exists primarily in documents whose very titles-e.g., "Toward Precision Medicine" -indicate their promissory nature. We disagree. PM is part of a longstanding attempt to reorient medical diagnosis and treatment to take advantage of genomics research and other approaches leveraging big data, such as electronic medical record research and crowd-sourced health tracking. These efforts are progressively elaborating an increasingly coherent vision of a different kind of medicine.,

As the prospects and challenges of PM loom before us, there ais urgent need to consider its implications for the social organization of medicine, particularly for the physician-patient relationship.

\section{A BASELINE MODEL OF THE PHYSICIAN-PATIENT RELATIONSHIP}

To explore how PM might change the physician-patient relationship, we compare it with an intentionally simplified baseline model drawn from prior work by sociologists: ${ }^{5,6}$

- To be sick is a dichotomous social role. Although natural realities are more ambiguous, society responds to illness by categorizing people as either healthy or sick. ${ }^{5}$ This categorization happens as a result of the doctor-patient interaction, and is aided by the use of cutoffs-formalized in checklists or internalized as expert heuristics-to impose clear order on uncertain diagnoses.
- The sick role anchors the individual in a social setting of care. To be sick is to be excused from the performance of certain social obligations and from responsibility for one's state. But exemption is granted only on the condition that one actively seeks help from people socially defined as qualified to care for illness. The patient is thus anchored in a familiar social script that involves an exchange of trust and care, governed by the goal of restoring normal functioning. ${ }^{5}$

- The physician-patient relationship is a gatekeeping process. The baseline model presumes that patients will approach physicians with a complaint. The physician's role is to manage the complaint - to link it to an objective finding, official diagnosis, and/or effective treatment; refer it; disconfirm it; or otherwise reach closure. The physician is the gatekeeper, controlling access to the status of being sick and to a social setting of care containing scarce resources. ${ }^{6,7}$

- The physician's authority rests on three pillars: the voluntary character of the sick role; the organization of medicine as a profession with a code of ethics and the authority to self-regulate; and the asymmetry of knowledge between physician and patient. ${ }^{5,7}$

\section{HOW WILL PM IMPACT THE PATIENT ROLE?}

The traditional sick role is likely to assume new and previously unexplored forms.

- Instead of one dichotomous role, there will be a multiplicity of hybrid statuses. PM replaces the traditional taxonomy of diseases with a multilayer characterization of individuals: information on genetic variants or other biomarkers, environments, and self-tracked lifestyle behaviors will be understood as risk factors for particular

${ }^{1}$ Precision Medicine and Society Program, Columbia University, New York, NY, USA. Correspondence: Paul S. Appelbaum (psa21@columbia.edu)

Submitted 31 May 2018; accepted: 16 August 2018

Published online: 14 September 2018 
conditions or as inputs to individual customization of care. $^{2-4,7,8}$ The persons thus characterized will no longer be either healthy or sick, but will occupy liminal spaces between the two poles. A good example is the phenomenon of "patients-in-waiting": when screening yields an abnormal finding, yet it is uncertain whether and when the individual will develop the condition. Such an individual may be closely monitored or given prophylactic treatment, thereby becoming a patient, although the "terms of exchange" of trust and care are unclear and constantly renegotiated. ${ }^{9}$ Moreover, family members who are carriers of mutations or only subclinically affected will be positioned in a similar hybrid status.

- Instead of anchoring patients in social settings of care, they and their families will be placed in increasingly uncertain situations. Social scientists who have observed the families of patients-in-waiting report high levels of confusion and stress. Families want to know whether their children are healthy or sick. An answer either way could anchor them in familiar institutional scripts. Yet ambiguous results regarding genetic status (e.g., in newborn screening) mean that clinicians are unable to provide straightforward answers, and instead vacillate between warning families to avoid overreaction or complacency. ${ }^{9}$ This exemplifies how PM can intensify the uncertainty of a previously stable medical encounter when the very goal of treatment-normal functioning-has become a moving target. It raises the possibility that instead of preventing diagnostic odysseys, PM will add a new type of "therapeutic odyssey."

- The sociopsychological burden of uncertainty will be shifted to patients. Paradoxically, the burden of uncertainty inherent in a probabilistic diagnosis will be increased by the expectation that the purportedly "precise" diagnosis will empower patients by giving them the opportunity to make better-informed decisions about future treatment. Patients will likely differ in their abilities to manage this tension due to inequalities of socioeconomic status, culture, age, and psychological disposition. $^{3}$

- Patients increasingly will surrender privacy to healthcare systems and information-sharing platforms. Along with information about patients' genomic status, healthcare systems and platforms will collect previously unavailable data about patients' environments and behaviors. Patients may be asked to record exercise, food intake, alcohol consumption, and mood for electronic transmission to their medical records. Passive monitoring of activity levels, geolocation, sleep patterns, and other behaviors will strip patients of the control they have had historically over whether to reveal such information to their physicians. They will have little control over how such data are aggregated, analyzed, and used., ${ }^{3,4}$

- The distinction between patient and research participant will be blurred. PM will create multiple situations in which treatment and research blend, as when diagnostic genome sequencing uncovers previously unknown pathogenic variants. In other situations, the very devices meant to individualize care (e.g., self-tracking apps) may double as platforms for the collection of research data. This raises concerns because researchers' ethical obligations fall short of physicians' fiduciary duties to patients, while participatory platforms often seek to monetize patients' data, and existing protections around experimental research may not apply. ${ }^{3}$

\section{HOW WILL PM IMPACT THE PHYSICIAN'S ROLE?}

Physicians too will see their roles evolve.

- Instead of managing patients' complaints, physicians will perform "bridging work." A process driven by screening requires the physician to fill the gap between abnormal results and patients' symptoms (or lack thereof), account for discrepancies, and decide whether the threshold for pathology has been met. The bridge can be built by reconceptualizing treatment as prevention of future illness, eliciting subclinical symptoms to confirm test results, or changing the cutoff for diagnosis. Doctors are currently ill-prepared for this task as they may lack the necessary genomic knowledge, and also because knowledge about the probable interactions among environmental, lifestyle, and -omic determinants of disease remains uncertain.

- Physicians' authority as gatekeepers will be diminished. Bridging work will be performed less by physicians alone and more as a negotiation among diverse parties: laboratories, administrators, regulatory agencies, payers, biomedical researchers, patient advocacy groups, and patients themselves. Doctors will "become part of a complex script that they cannot alter or opt out from without incurring excessive transaction costs." ${ }^{3}$ Contrary to prescriptive accounts of "patient centeredness," we do not believe that PM is likely to transfer sole gatekeeping authority to patients and their families. Rather, both patients and physicians are at risk of losing control over the gatekeeping function, as the "datafication" of health care evokes increased levels of surveillance by health-care systems and information platforms, where data are integrated and analyzed. ${ }^{3}$ With the decline in gatekeeping authority, physicians will find it harder to secure patients' trust and cooperation unless they undertake to act as their advocates.

- Instead of a singular patient-physician asymmetry of knowledge, physicians will need to adapt to new information asymmetries. Because many physicians report discomfort in interpreting genomic results, ${ }^{8}$ and do not possess the skills to analyze the huge data sets created by "datafication," informational and interpretive dominance will shift to health-care and data platform managers, specialists, laboratories, and patient advocacy groups. ${ }^{3}$ That trend is likely to increase as more "-omic" 
data come online for clinical application. Yet, if existing information asymmetries are not to be further exacerbated, especially for patients in low-resourced settings, physicians must be trained to mediate the disclosure of genomic and other results from $\mathrm{PM}$ approaches to patients. ${ }^{10}$

Assuming the accuracy of this forecast, medical education and training-including continuing education-cannot begin soon enough to prepare the profession for these changes. Teaching genetics will not be enough. Physicians must be prepared to help patients deal with an onslaught of unfamiliar information, from multiple -omic and environmental sources, often with uncertain clinical implications, while striving to treat the patient and not the laboratory findings or the virtual "data double" of the patient. $3,4,7$ They must learn to convey complex information to patients and families in understandable terms, with due attention to patients' emotional needs, tolerance for decision making under conditions of ambiguity, and shared ownership of uncertainty. Precision medicine, for all its futuristic promise, will ultimately depend on some of the oldest medical skills.

\section{DISCLOSURE}

The authors declare no conflicts of interest.

\section{REFERENCES}

1. Juengst $E$, et al. From 'personalized' to 'precision' medicine: the ethical and social implications of rhetorical reform in genomic medicine. Hastings Cent Rep. 2016;46:21-33.

2. Committee on a Framework for Development of a New Taxonomy for Disease, National Research Council. Toward precision medicine: building a knowledge network for biomedical research and a new taxonomy of disease. Washington, DC: National Academies Press; 2011.

3. Prainsack B. Personalized medicine: empowered patients in the $21 \mathrm{st}$ century? New York: NYU Press; 2017.

4. Cribb A. Healthcare in transition.. Bristol, UK: Policy Press; 2017.

5. Parsons T. Illness and the role of the physician. Am J Orthopsychiatry. 1951;21:452-460.

6. Goffman E. The moral career of the mental patient. In: Goffman E, ed. Asylums: essays on the social situation of mental patients and other inmates. New York: Anchor Books; 1961:125-170

7. Berwick D. What 'patient-centered' should mean: confessions of an extremist. Health Affairs. 2009;28:w555-w565.

8. Topol E. The creative destruction of medicine: how the digital revolution will create better health care. New York: Basic Books; 2012.

9. Timmermans $S$, Buchbinder $M$. Saving babies? The consequences of newborn genetic screening. Chicago: University of Chicago Press; 2013

10. Sabatello M, Appelbaum P. The precision medicine nation. Hastings Cent Rep. 2017;47:19-29. 